Research Article

\title{
Pattern Recognition and Neural Network-Driven Roller Track Analysis via 5G Network
}

\author{
Yuliang Guo iD \\ Dalian Maritime University, Dalian 116026, China \\ Correspondence should be addressed to Yuliang Guo; guoyl1975@dlmu.edu.cn
}

Received 19 November 2020; Revised 1 December 2020; Accepted 16 December 2020; Published 30 December 2020

Academic Editor: Jianhui Lv

Copyright ( 92020 Yuliang Guo. This is an open access article distributed under the Creative Commons Attribution License, which permits unrestricted use, distribution, and reproduction in any medium, provided the original work is properly cited.

Roller skating is an important and international physical exercise, which has beautiful body movements to be watched. However, the falling of roller athletes also happens frequently. Upon the roller athletes' fall, it means that the whole competition is over and even the roller athletes are perhaps injured. In order to stave off the tragedy, the roller track can be analyzed and be notified the roller athlete to terminate the competition. With such consideration, this paper analyzes the roller track by using two advanced technologies, i.e., pattern recognition and neural network, in which each roller athlete is equipped with an automatic movement identifier (AMI). Meanwhile, AMI is connected with the remote video monitor referee via the transmission of $5 \mathrm{G}$ network. In terms of AMI, its function is realized by pattern recognition, including data collection module, data processing module, and data storage module. Among them, the data storage module considers the data classification based on roller track. In addition, the neural network is used to train the roller tracks stored at AMI and give the further analysis results for the remote video monitor referee. Based on NS3, the devised AMI is simulated and the experimental results reveal that the prediction accuracy can reach $100 \%$ and the analyzed results can be used for the falling prevention timely.

\section{Introduction}

With respect to the beautiful sports, the roller skating is a nonnegligible and international physical exercise thanks to its graceful body movements. Since the roller athletes make the highly ornamental postures during the process of competition, it perhaps causes the dangerous situations, where the falling of roller athletes is regarded as the most representative tragedy $[1,2]$. Upon the roller athletes' fails, this indicates that the whole roller competition is forced to be over; at the same time, the roller athletes may be seriously affected. Therefore, it is a very important research topic to make the falling detection for the roller athletes. Regarding this, a comment and popular method is to analyze the roller track, and based on this, the falling risk can be decreased.

The neural network [3-5] is usually used to analyze the behavior track including but not limited to the roller track, this is because it has some obvious advantages. For example, it has the strong computation ability, especially for the largescale data features. Besides, it also has the strong stability to obtain the convergent analysis results. To sum up, the neural network can be used to train the prestored data related to the previous roller tracks and give the real-time training results to be used for the falling prevention. Well, there are a number of neural network models, but the back-propagation (BP) neural network [6, 7] has the simplest network structure. Thus, this paper uses BP neural network to do the data training.

In spite of this, it also exits three issues: at first, where the BP neural network module is embedded; then, what the prestored data container is; finally, how to transmit the analysis result between roller athletes and the remote video monitor referee. These first two issues require this paper to explore an additional component, i.e., automatic movement identifier, (AMI) to store such roller tracks data. In particular, AMI is equipped into the body of roller athlete. In other words, the BP neural network module is embedded into AMI and used to train the roller tracks stored at AMI. However, the further problem is how to obtain the roller tracks data. Regarding this, the pattern recognition $[8-10]$ is 
a highly-regarded candidate. That is to say, the function of AMI can be realized by pattern recognition by three inherent modules, i.e., data collection, data processing, and data storage. Furthermore, in terms of the last issue, it has the ultra-high requirement on the delay and stability of data transmission. Upon the transmission delay being out of the certain level of acceptance, the falling behavior cannot be avoided even though the system has detected the analysis results on the falling of roller athletes. Similarly, if the data transmission is unstable, the remote video monitor referee and roller athletes cannot make the following decision well. With these concerns, the 5G-enabled network can be deployed at the competition gymnasiums and stadiums so as to enhance the transmission stability and cut down the transmission delay.

By reviewing the above statements, it is observed that pattern recognition and $\mathrm{BP}$ neural network are used to analyze the roller track, and the $5 \mathrm{G}$ network is used to guarantee the data transmission [11-13]. To sum up, the contributions of this paper are shown as follows. At first, the whole roller track analysis structure is introduced. Then, AMI is devised and its function is realized by pattern recognition. Thirdly, the improved BP neural network is used to do the data training. Finally, the experimental results reveal that the prediction accuracy can reach $100 \%$ and the analyzed results can be used for the falling prevention timely.

The remaining paper is organized as follows. Section 2 introduces the whole network architecture. Section 3 uses pattern recognition to realize AMI. Section 4 uses BP neural network to train the data. Section 5 reports the experimental results. The overview of this paper is concluded in Section 6 .

\section{Network Architecture}

The whole network architecture includes two character roles, i.e., roller athlete and remote video monitor referee, where the former is the main research object and the latter is the assistant research optimization, as shown in Figure 1. In particular, the whole network architecture introduces a new component, i.e., AMI to store the roller tracks data, and AMI is equipped into the body of roller athlete.

Furthermore, AMI consists of two modules, i.e., pattern recognition and BP neural network. Meanwhile, the pattern recognition module is used to realize AMI based on data collection, data processing, and data storage, where the data storage part considers the data classification based on roller track. The BP neural network module is used to train the roller tracks stored at AMI and give the further analysis results.

Moreover, based on the analyzed results, the whole network architecture involves two kinds of message transmissions. On one hand, AMI delivers the analyzed results to the roller athlete directly and the competition is forced to be terminated. On the other hand, AMI delivers the analyzed results to the remote video monitor referee for conducting the posture adjustment. If the analyzed results belong to the emergency situation, the first kind of message is performed; otherwise, the second kind of message is performed. Especially for the second condition, the data transmission

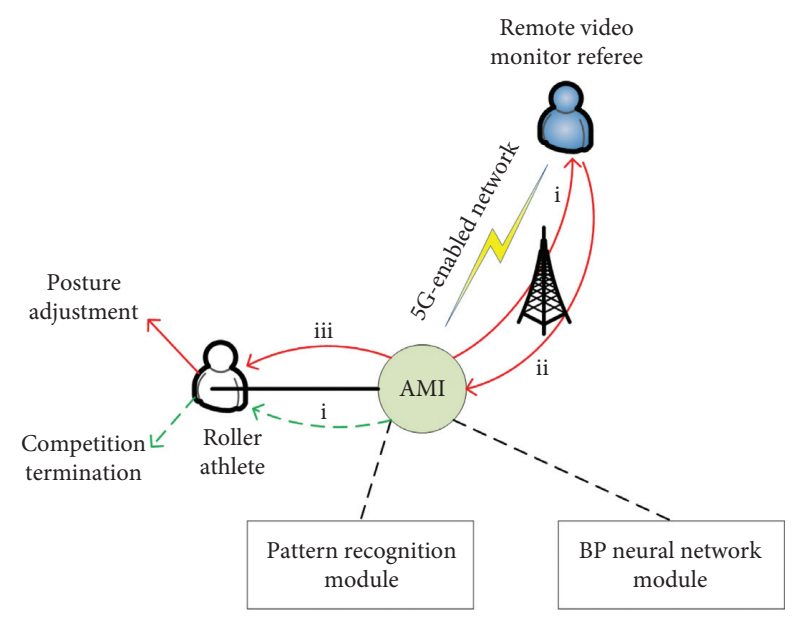

FIgURE 1: The whole network architecture.

between AMI and remote video monitor referee depends on the 5G-enabled network to guarantee the fast and stable performance.

\section{Pattern Recognition}

The pattern recognition is used to realize the function of AMI, which includes data collection, data processing, and data storage. The system framework of pattern recognition is shown in Figure 2, and the whole process is completed based on field-programmable gate array (FPGA) [14]. To be specific, the inherent static random access memory (SRAM) [15] is used to extract the roller tracks. Then, the digital signals are converted into the analog signals by a D/A transverter. Finally, the analog signals are amplified based on rejector and input into FPGA for receiving, processing, and storing.

3.1. Feature Vector Determination. During the process of data classification for roller tracks, it involves the attribution problem in terms of data samples. For such problem, the basic principle is to build the maximum membership degree function. Furthermore, let $W_{x}$ denote the $x$-th data object's feature vector, and for $N$ data applications in terms of roller tracks, $W_{x}$ is expressed as $\left\{w_{1}, w_{2}, \ldots, w_{N}\right\}$ and $W_{x} \in f(g)$. For any $g_{0} \in G$, if it exits, $i \leq N$, and $g_{0}$ is regarded as one membership degree value of $W_{x}$. On this basis, all membership degree functions can be determined based on the feature values of roller tracks, which are used to make the data classification of roller tracks.

Regarding the determination of feature vector, the law of large numbers based on the Chebyshev [16] is used to obtain the coagulation equation with respect to the random variable:

$$
\left[\frac{h-G[h]}{G[h]}<\delta\right] \geq 1-\frac{\operatorname{var}[h]}{\delta G^{2}[h]},
$$

where $h$ is a random variable; var $[h]$ is the variance; $G[h]$ is the average value; and $\delta$ is the error value. When the variance is relatively small, the corresponding error value is smaller 


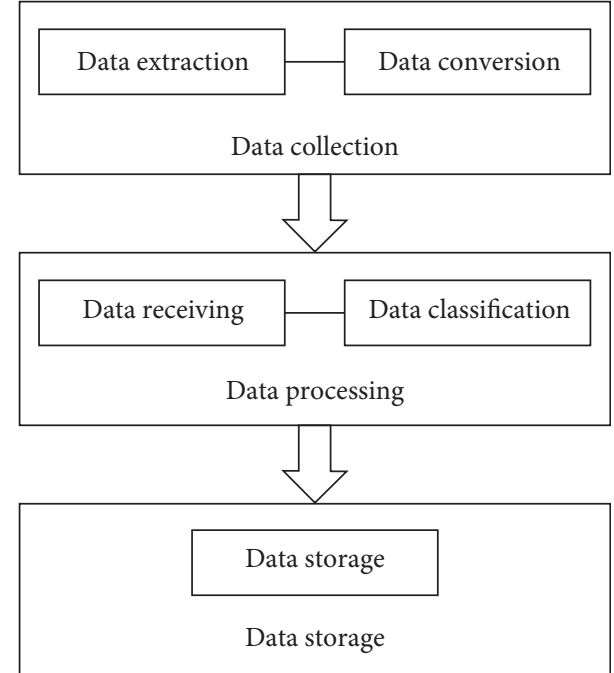

Figure 2: The whole system framework of pattern recognition.

than $\delta$. In other words, when and only when the coagulation value is around the average value, the relatively small error value can be guaranteed. In order to further make the data classification for roller tracks, this paper introduces a resolution for objective set:

$$
P=\frac{\left|G\left[h_{0}\right]-G\left[h_{a}\right]\right|}{\sqrt{\operatorname{var}\left[h_{0}\right]}+\sqrt{\operatorname{var}\left[h_{a}\right]}},
$$

where $h_{0}$ is the object and $h_{a}$ is the backdrop.

3.2. Membership Degree Function Computation. Let $V_{i}$ denote any data application's feature vector, i.e., $V_{i}=\left\{v_{1}, v_{2}, \ldots, v_{j}\right\}$, where $v_{j}$ is the subcomponent of $V_{i}$ and it concentrates around the average value. For a data application $a, v_{j}$ is used to compute the related membership degree value $e_{a}$. In particular, the Cauchy distribution [17] is used as the reference of the membership degree function, as shown in Figure 3.

Based on the Cauchy distribution reference, $e_{a}$ is computed as follows:

$$
e_{a}=1-\frac{\left|v_{j}-\mathrm{ave}\right|}{\sum_{N} v_{j}-\mathrm{ave}}
$$

where ave is the average value. Regarding further obtaining of the method of data storage in terms of AMI, this paper uses the maximum membership degree to address this. Let $D_{a}$ denote the maximum membership degree function regarding $a$, and it is defined as follows:

$$
D_{a}=\Delta e_{a}, \quad a \in[1, j] .
$$

According to $D_{a}$, this paper can complete the data storage in AMI by considering the data classification based on roller tracks, and the related pseudocode is described as follows. Among them, line 1 means the clocks collection; line 2 means the initialization of serial communication clocks; lines 4-5 and 7-8 mean the address processing; line 6 means

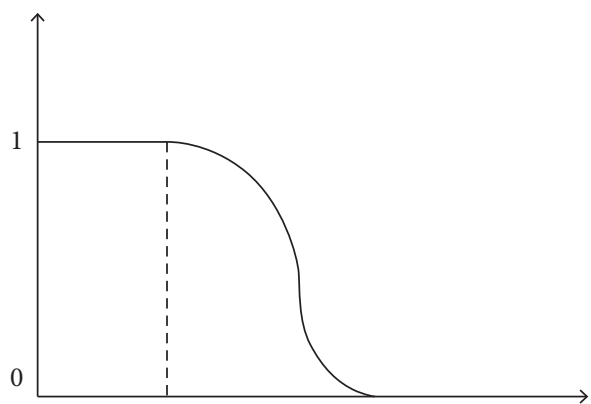

FIgure 3: The Cauchy distribution reference.

to output the handled data; line 9 means the completion of data storage.

(1) module flash

(2) input w_clk

(3) input $r_{-}$clk

(4) input w_runtrack

(5) input r_runtrack

(6) output $[8: 0]$ datain

(7) input $[9: 0]$ wrads

(8) input $[9: 0] \mathrm{rads}$

(9) input $[8: 0]$ set_rtrack

\section{BP Neural Network}

BP neural network is a feedforward network with the multiple layers, and its topology is shown in Figure 4, including $n$ input layers, $p$ hidden layers, and $q$ output layers.

For the BP neural network, the weight adjustment is considerably important, which is as follows:

$$
\begin{gathered}
\Delta w_{j, k}=-\eta \frac{\partial E}{\partial w_{j, k}}=\eta \delta_{k} y_{j}, \\
\Delta w_{i, j}=-\eta \frac{\partial E}{\partial w_{i, j}}=\eta \delta_{j} x_{i},
\end{gathered}
$$

where $w_{j, k}$ is the network weight between the output layer and hidden layer; $w_{i, j}$ is the network weight between the input layer and hidden layer; $\Delta w_{j, k}$ and $\Delta w_{i, j}$ are two increments with respect to the weight; $x_{i}$ is the input data from the $i$-th node; $y_{j}$ is the output data from the $j$-th node; and $\eta \in(0,1)$ is the learning rate of BP neural network. In particular, the negative sign means the gradient descent action. Furthermore, $E$ is the error function of BP neural network, and it is defined as follows:

$$
E=\frac{1}{2} \sum_{o=1}^{q}\left(d_{o}(n)-y_{o}(n)\right)^{2},
$$

where $d_{o}(n)$ is the expected output result and $y_{o}(n)$ is the actual output result.

However, the traditional BP neural network has two obvious limitations. On one hand, the training speed is very slow; on the other hand, it is very difficult to converge to the 


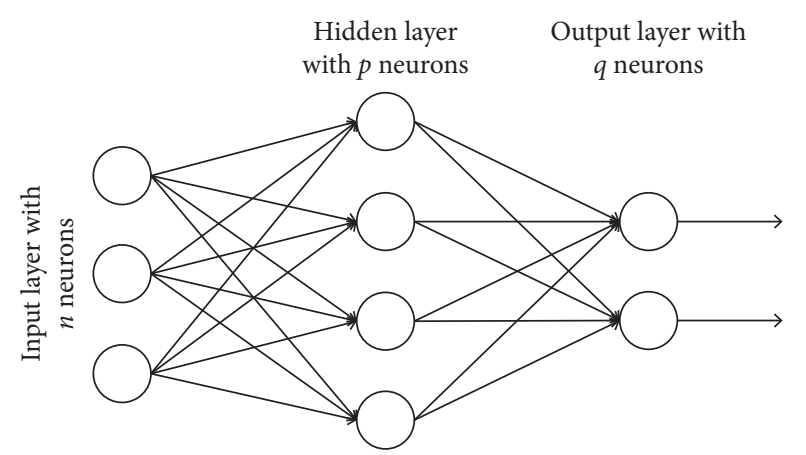

FIGURE 4: BP neural network topology.

global optimum. Therefore, in order to enhance the recognition efficiency and the accurate rate of roller track analysis, this paper improves the traditional BP neural network from two aspects, i.e., momentum attachment and learning rate optimization.

By using the momentum attachment method, the improved weight adjustment is as follows:

$$
\Delta w(I+1)=\beta(w(I+1)-w(I))+\alpha(1-\beta) \frac{\partial E(I)}{\partial w(I)},
$$

where $\beta$ is the momentum factor; $\alpha$ is the self-learning rate; $I$ is the number of training; and $(\partial E(I) / \partial w(I))$ is the gradient of $w(I)$. Equation (7) indicates that, when the modeling error decreases, the different momentum factors are used to adjust the whole network parameters so as to reach the global optimum in terms of the network performance. To be specific, when the surface of error is the flat state, the momentum factor is increased to accelerate the convergence process; on the contrary, when the surface of error is the steep state, the momentum factor is decreased to avoid the network instability. According to the abovementioned statements, the momentum factor is defined as follows:

$$
\beta=e^{-R-\|\partial E(I) / \partial w(I)\|},
$$

where $R$ is a random number, satisfying $R \in(0,1)$. In particular, when the surface of error is the flat state and the corresponding curve stays the bottom, $(\partial E(I) / \partial w(I))$ gradually decreases until the following equation is satisfied:

$$
\Delta w(I+1)=\beta(w(I+1)-w(I))=\Delta w(I),
$$

which avoids the situation of $\Delta w(I)=0$.

In fact, the unchanging learning rate has the important influence on the BP neural network performance. In order to guarantee that the BP neural network always has the maximal training effect, the dynamic learning rate is defined as follows:

$$
\eta(I)=\left\{\begin{array}{l}
\alpha(I-1), \quad E(I)<E(I-1), \\
\alpha(I-1) e^{-\gamma}, \quad \text { otherwise, }
\end{array}\right.
$$

where $\gamma$ is a parameter, satisfying $\gamma \in[0.0001,0.001]$.

According to the abovementioned statements, the BP neural network is used to train the roller tracks stored at AMI, and the related pseudocode is described as follows:
(1) Input the roller tracks data

(2) Build BP neural network according to Figure 4

(3) Add momentum factor $\beta$ and self-learning rate $\alpha$ into BP neural network

(4) Compute the error function according to equation (6)

(5) Modify the weight adjustment

(6) Check whether the number of training satisfies the given $I$

(7) Output the analyzed results on roller tracks

(8) Send the results to the roller athlete or the monitor referee according to the real emergency

\section{Performance Evaluation}

The proposed pattern recognition and neural networkdriven roller track analysis method, called PRNN, is implemented by NS3 [18], which is a discrete event emulator. Since the open dataset on the roller tracks cannot be obtained, this paper simulates 1500 roller competitions and considers them as the data trace. In addition, this paper is the first paper to propose roller track analysis based on the emerging computer technologies, and there is no suitable comparison baseline. As a result, this paper considers the method without the improved BP neural network, called PRWI, as the baseline. Meanwhile, there are four $5 \mathrm{G}$ wireless base stations which are used to support the efficient data communication. The detailed deployment is shown in Figure 5 .

5.1. Parameter Determination: $\alpha$ and $\beta$. For the improved BP neural network, $\alpha$ and $\beta$ are two important parameters. In this paper, suppose that $\alpha, \beta=\{0.1,0.2,0.3,0.4,0.5,0.6$, $0.7,0.8,0.9\}$, and different settings generate different network performances. In this section, the standard deviation is used to measure the network performance, and the smaller standard deviation means the better network performance. The experimental results on standard deviation with respect to different $\alpha$ and $\beta$ settings are shown in Table 1 and Figure 6.

As can be seen from Table 1 and Figure 6, with the increasing of $\alpha$, the change of standard deviation includes two phases. At first, the standard deviation gradually decreases. Then, when $\alpha$ reaches 0.7 , the standard deviation will have the minimal value. After that, the standard deviation gradually increases. As a result, $\alpha$ is set as 0.7 . Different from $\alpha$, the standard deviation from $\beta$ shows fours change phases, and the corresponding inflection points are $0.4115(\beta=0.3)$, $0.5106(\beta=0.6)$, and $0.4953(\beta=0.7)$ respectively. It is obvious that when $\beta=0.3$, the corresponding standard deviation has the minimal value. As a result, $\beta$ is set as 0.3 .

5.2. Prediction Ratio. Based on $\alpha=0.7$ and $\beta=0.3$, this paper performs the roller track analysis based on 1500 roller competitions, where the number of simulations is set as 15 . The experimental results on prediction ratio with respect to 


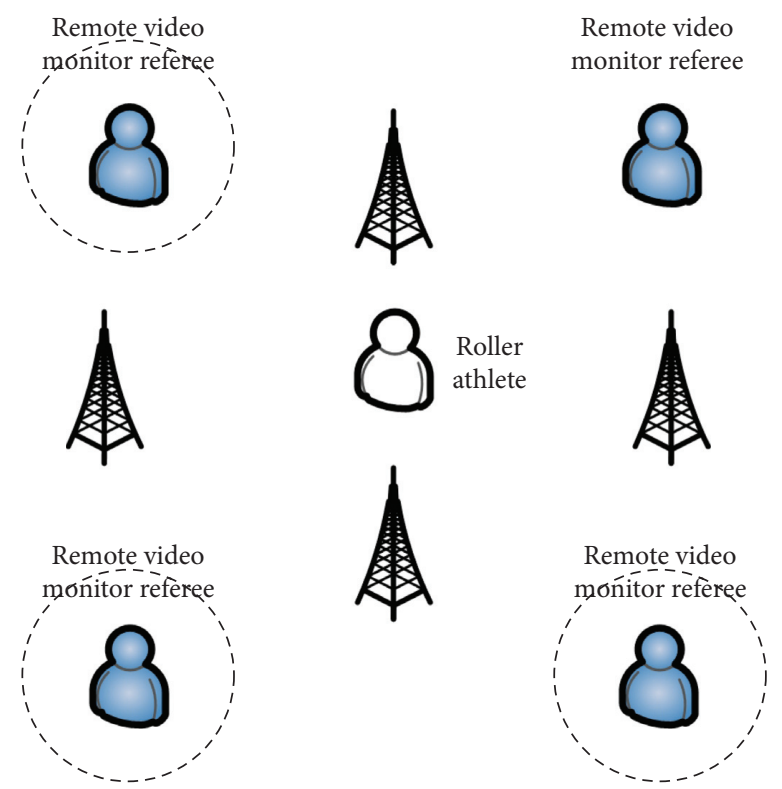

Figure 5: The detailed deployment on 5G-enabled network.

TABLE 1: The experimental results on standard deviation with respect to different $\alpha$ and $\beta$ settings.

\begin{tabular}{lccccccccc}
\hline$\alpha$ & 0.1 & 0.2 & 0.3 & 0.4 & 0.5 & 0.6 & 0.7 & 0.8 & 0.9 \\
\hline Standard deviation & 0.6323 & 0.5964 & 0.5319 & 0.5216 & 0.4906 & 0.4534 & $\mathbf{0 . 3 6 1 3}$ & 0.4059 & 0.4638 \\
$\beta$ & 0.1 & 0.2 & 0.3 & 0.4 & 0.5 & 0.6 & 0.7 & 0.8 & 0.9 \\
Standard deviation & 0.5361 & 0.4937 & $\mathbf{0 . 4 1 1 5}$ & 0.4661 & 0.5032 & 0.5106 & 0.4953 & 0.5357 & 0.5661 \\
\hline
\end{tabular}

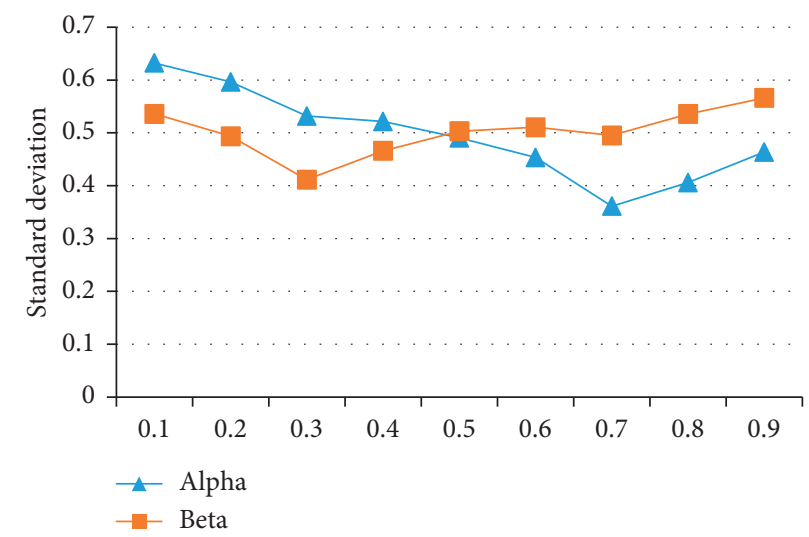

Figure 6: Different standard deviations with different $\alpha$ and $\beta$ settings.

different simulations are shown in Figure 7. It is observed that PRNN always has better prediction ratio than PRWI, and the prediction ratio of PRNN always keeps $100 \%$, because this paper enhances the traditional BP neural network from two aspects, i.e., momentum attachment and learning rate optimization. For PRWI, its prediction ratio is low and the corresponding stability is also bad, varying from $86.49 \%$ (the ninth simulation) to $95.06 \%$ (the second simulation).

5.3. Communication Time. Although PRNN has reached $100 \%$ prediction ratio, this does not mean that the analyzed results can be used for the falling prevention timely. Therefore, this section tests the communication time to illustrate the availability of PRNN. For 1500 roller competitions, there are 60 times events requiring AMI to transmit the adjustment message or the termination message. Furthermore, there are 54 times events for the first condition while there are 6 times events for the second condition. The communication time between AMI to the roller athlete can be neglected due to the fact that AMI is equipped into the body of roller athlete. Given this, this paper only tests the communication time between AMI and the remote video monitor referee. The average communication times regarding 54 times events under different simulations are shown in Figures 8-11, where Figure 8 shows the experiment results based on 1 Gbps network bandwidth, Figure 9 shows 


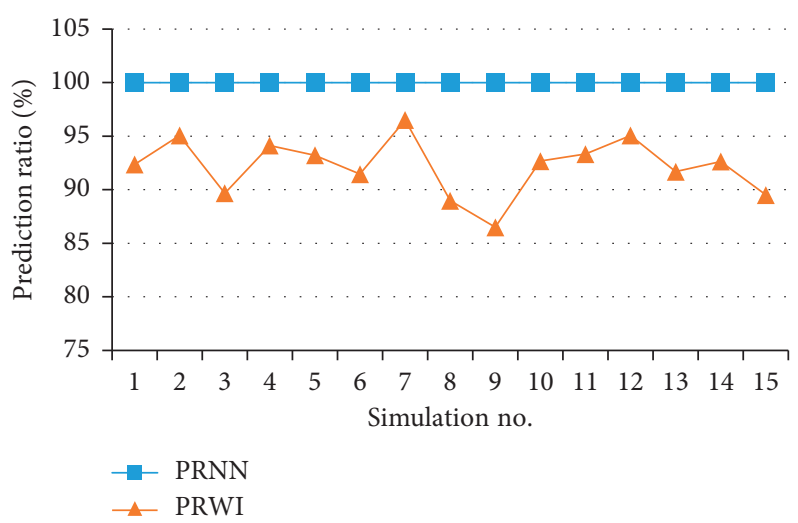

FIgURE 7: The experimental results on prediction ratio with respect to different simulations.

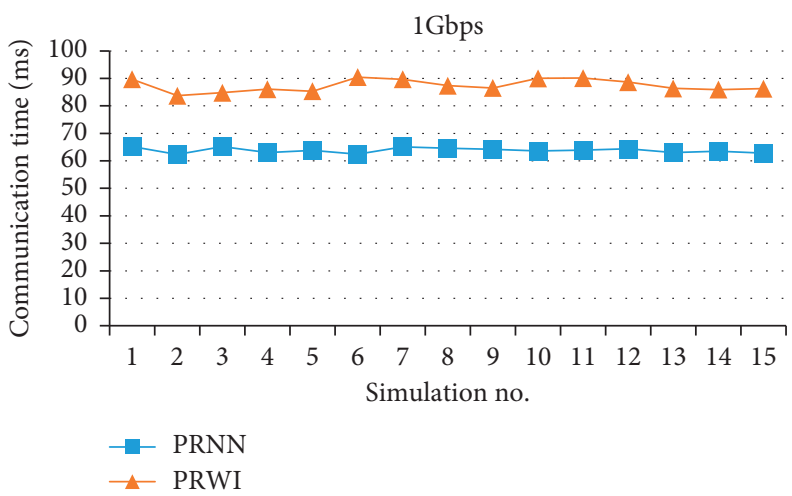

FIgURE 8: The experimental results on communication time with respect to different simulations, where network bandwidth is 1 Gbps.

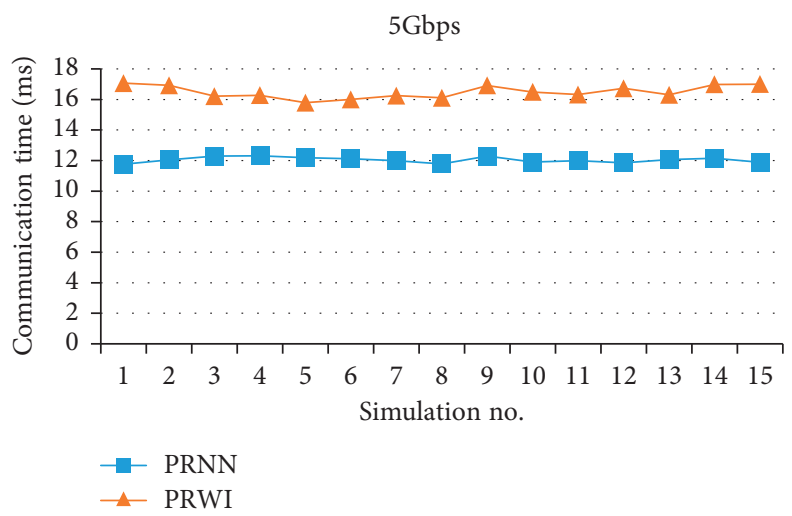

FIGURE 9: The experimental results on communication time with respect to different simulations, where network bandwidth is 5 Gbps.

those based on 5 Gbps network bandwidth, Figure 10 shows those based on 10 Gbps network bandwidth, and Figure 11 shows those based on 15 Gbps network bandwidth.

As can be seen from Figures 8-11, the communication time of PRNN is always smaller than that of PRWI, this is because the improved BP neural network accelerates the convergence speed. Furthermore, under the scenario of 5Genabled network, with the increasing of network bandwidth, the communication time decreases drastically. It suggests that $5 \mathrm{G}$ network has great performance improvement in terms of the communication time. In addition, it is also observed that, with the increasing of network bandwidth, the communication time difference between PRNN and PRWI becomes smaller and smaller. This suggests that $5 \mathrm{G}$ network can guarantee the availability of PRNN; that is to say, the analyzed results can be used for the falling prevention timely. 


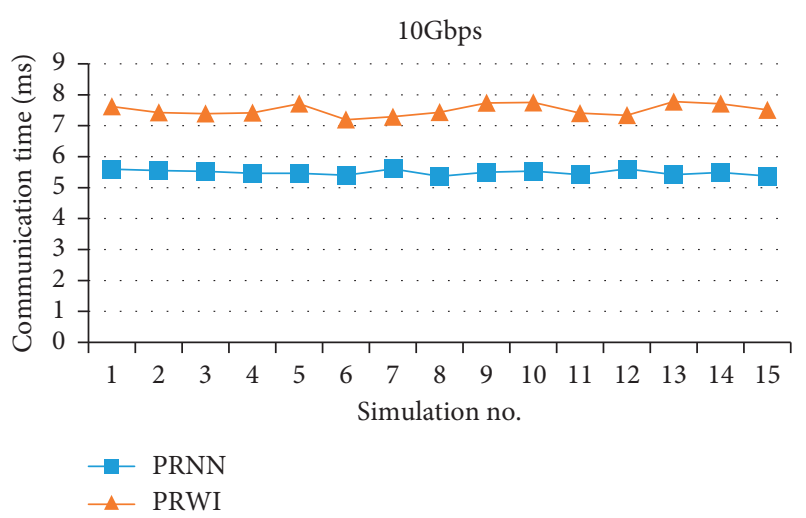

FIGURE 10: The experimental results on communication time with respect to different simulations, where network bandwidth is 10 Gbps.

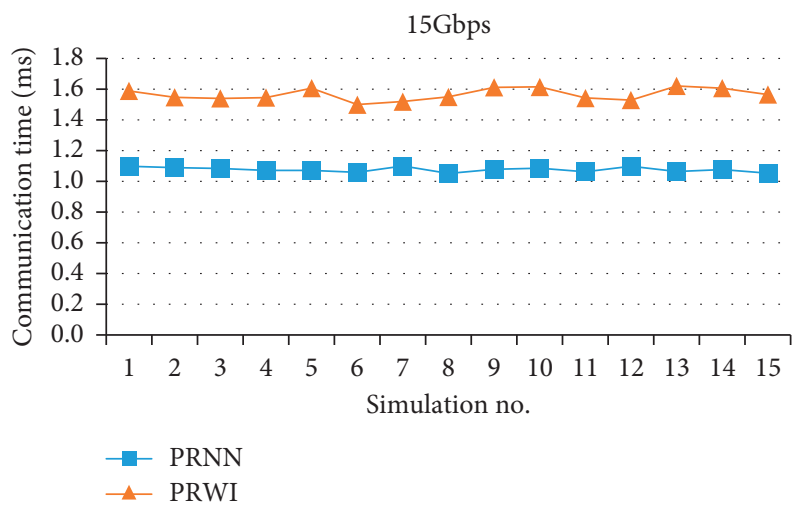

FIGURE 11: The experimental results on communication time with respect to different simulations, where network bandwidth is 15 Gbps.

\section{Conclusions}

This paper studies the roller track analysis based on pattern recognition and $\mathrm{BP}$ neural network. In particular, the 5Genabled network is used to guarantee the stability and efficiency of transmission. Meanwhile, regarding pattern recognition, it is used to realize the function of AMI, which includes data collection, data processing, and data storage, and the whole process is completed based on FPGA. Then, regarding BP neural network, this paper improves it by considering momentum attachment and dynamic learning rate. The simulation is driven by NS3, where prediction ratio and communication time are regarded as two performance evaluation metrics, and the experimental results demonstrate that the proposed roller track analysis method can be used for the falling prevention of roller athlete timely.

This paper is the first paper to propose roller track analysis based on the emerging computer technologies, which has the important reference value in the field of sports. However, this paper also has some limitations. At first, the system platform of AMI is not implemented. Then, the simulation only depends on NS3 and lacks of the verification based on the test-bed. At last, the posture variety of roller athlete is not considered. In future, the abovementioned limitations will be overcome.

\section{Data Availability}

The data used to support the findings of this study are available from the corresponding author upon request.

\section{Conflicts of Interest}

The author has no conflicts of interest.

\section{References}

[1] Q. Bu, S. Lan, C. Gu et al., "A judgment framework for roller skating using faster R-CNN," in Proceedings of the 2017 International Conference on the Frontiers and Advances in Data Science (FADS), pp. 53-57, Xi'an, China, October 2017.

[2] G. Olmi, "Failure of the chassis of roller skates for agonistic figure skating," Case Studies in Engineering Failure Analysis, vol. 3, pp. 62-67, 2015.

[3] L. Ma, S. Cheng, and Y. Shi, "Enhancing learning efficiency of brain storm optimization via orthogonal learning design," IEEE Transactions on Systems, Man, and Cybernetics: Systems, 2020, In press.

[4] P. Suresh Kumar, H. S. Behera, A. Kumari et al., "Advancement from neural networks to deep learning in software effort estimation: perspective of two decades," Computer Science Review, vol. 38, pp. 1-32, 2020.

[5] S. Kaviani and I. Sohn, "Influence of random topology in artificial neural networks: a survey," ICT Express, vol. 6, no. 2, pp. 145-150, 2020.

[6] Z. Xu, R. Zhang, and W. Jing, "When does online BP training converge?" IEEE Transactions on Neural Networks, vol. 20, no. 10, pp. 1529-1539, 2009.

[7] W. Wu, G. Feng, Z. Li, and Y. Xu, "Deterministic convergence of an online gradient method for BP neural networks," IEEE Transactions on Neural Networks, vol. 16, no. 3, pp. 533-540, 2005.

[8] M. Paolanti and E. Frontoni, "Multidisciplinary pattern recognition applications: a review," Computer Science Review, vol. 37, pp. 1-23, 2020.

[9] X.-Y. Zhang, C.-L. Liu, and C. Y. Suen, "Towards robust pattern recognition: a review," Proceedings of the IEEE, vol. 108, no. 6, pp. 894-922, 2020.

[10] V. Jacobson, J. J. Li, K. Tapia et al., "Visualizing neural networks for pattern recognition," in Proceedings of the International Conference on Pattern Recognition and Artificial Intelligence-PRAI 2018, pp. 18-22, Union, NJ, USA, August 2018.

[11] F. Moggio, M. Boldi, S. Canale et al., "5G EVE a european platform for 5G application deployment," in Proceedings of the 14th International Workshop on Wireless Network Testbeds, Experimental Evaluation \& Characterization, pp. 124-125, London, UK, September 2020.

[12] A. Jain, E. Lopez-Aguilera, and I. Demirkol, "Are mobility management solutions ready for $5 \mathrm{G}$ and beyond?" Computer Communications, vol. 161, pp. 50-75, 2020.

[13] J. Navarro-Ortiz, P. Romero-Diaz, S. Sendra, P. Ameigeiras, J. J. Ramos-Munoz, and J. M. Lopez-Soler, "A survey on 5G usage scenarios and traffic models," IEEE Communications Surveys \& Tutorials, vol. 22, no. 2, pp. 905-929, 2020.

[14] A. G. Blaiech, K. Ben Khalifa, C. Valderrama, M. A. C. Fernandes, and M. H. Bedoui, "A survey and 
taxonomy of FPGA-based deep learning accelerators," Journal of Systems Architecture, vol. 98, pp. 331-345, 2019.

[15] P. Cascaval and D. Cascaval, "March test algorithm for unlinked static reduced three-cell coupling faults in randomaccess memories," Microelectronics Journal, vol. 93, pp. 1-4, 2019.

[16] B. K. Muite, "A numerical comparison of Chebyshev methods for solving fourth order semilinear initial boundary value problems," Journal of Computational and Applied Mathematics, vol. 234, no. 2, pp. 317-342, 2010.

[17] X. Wang, J. Lv, M. Huang et al., "Energy-efficient ICN routing mechanism with QoS support," Computer Networks, vol. 131, pp. 38-51, 2018.

[18] NS3, https://www.nsnam.org. 